\title{
4
}

\section{Virtual reality in manufacturing}

\author{
Dr. A. K. Bejczy \\ Jet Propulsion Laboratory, MS198-219 \\ California Institute of Technology \\ Pasadena, CA 91109-8099 \\ Phone: (818) 354-4568 \\ Fax: (818) 393-5007 \\ e-mail: bejczy@telerobotics.jpl.nasa.gov
}

\begin{abstract}
Computer graphics based virtual reality techniques offer powerful and very valuable task visualization aids for planning system set-up and system processes, for previewing system and device actions, and even for seeing some invisible things in a complex action or production environment. Virtual reality techniques connected to CAD data banks can also offer 3-D simulation based programming and control tools for computer-controlled devices and can provide real-time interactive operator interface to those devices. Virtual reality techniques can also be applied to quick product prototyping by high-fidelity product modeling
\end{abstract}

\section{Keywords}

Computer graphics, visualization, modeling, operator interface, computer programming and control.

\section{INTRODUCTION}

Visualization is a key problem in many areas of science and engineering. The emerging 'virtual reality' technology, which is a major technical idea and undertaking of the 1990s, can help resolve or lessen many visualization problems.

The term 'virtual reality' (VR) essentially denotes the creation of realistic looking world scenarios by the use of computer graphics simulation techniques which in turn can be connected to Computer Aided Design (CAD) data banks. A distinctive feature of VR products is that the synthetic world scenarios are not static but dynamic: the user can interact with them in various ways in real time. 
VR technology already found its way in many application areas like entertainment, advertising, arts, medicine, robotics, and lately also, in manufacturing. This article is focused at applications or application potential of VR in manufacturing. In the first part of the article actual robotic/telerobotic application examples are quoted to illustrate the application potential of VR in manufacturing, since robotics to a large extent is connected to manufacturing. The second part of the article specifically addresses manufacturing topics.

\section{VIRTUAL REALITY IN ROBOTICS}

The role of VR in robotics includes (i) task visualization for planning system set-up and actions, (ii) action preview prior to actual motion execution, (iii) predicting motions in real time in telerobotics when there is a considerable communication time delay between operator control station and remote robot, (iv) operator training prior to exercising hardware for learning system operation, (v) enabling visual perception of non-visible conditions and events like multi-dimensional workspace constraints, hidden proximity motion states and actual contacts, (vi) constructing virtual sensors for training and for safety under actual operations, and (vii) to serve as a flexible operator interface modality replacing complex switchboard and analog display hardware.

\section{Dual-arm workcell}

A VR system was developed for a dual-arm workcell of the Advanced TeleOperation Project (ATOP) at the Jet Propulsion Laboratory (JPL) a few years ago. The workcell was surrounded by a gantry robot frame providing two-d.o.f. mobility for a stereo and two mono TV cameras, each on a pan-tilt base, in three orthogonal planes. Camera focus, zoom, and iris can also be controlled remotely. The workcell housed two eight-d.o.f. robot arms, each equipped with a 'smart' robot claw-hand which can sense both the grasp force at the base of the claws and the three orthogonal forces/moments at the base of the hand. The task environment in the workcell simulated the Solar Maximum Satellite Repair (SMSR) which was actually performed by two astronauts in space suits in the Space Shuttle cargo bay in 1983. (Note that this satellite was not designed for repair!). The goal of the experiments in the ATOP workcell was to show how to do the same repair in a teleoperation mode. To achieve this goal, the use of computer graphics offered an indispensable help in many ways.

Altogether, twelve major sequential subtasks are implied in the Main Electrical Box (MEB) change-out in the SMSR mission, and each subtask requires the use of some tool (screwdriver, scissors, etc.) This corresponds to a large set of task and control visualization off-line analysis by VR techniques, also taking account of workspace, arm kinematics, use of tools, and viewing constraints. It is noted that the motion of the robot arms' graphics images during task and control simulation are controlled by the same inverse/forward kinematics control software that controls the actual hardware system.

Figure 1 illustrates a graphics operator interface to the control of the dual-arm telerobot system for SMSR experiments, as a result of the off-line VR task and control simulation 
analysis. The subtasks are listed in the lower right insert, four preview control options are listed in the upper left insert, and the upper right insert lists some current status messages. It is noted that the graphics image of the two arms is updated in real time at a rate of at least $15 \mathrm{~Hz}$ so that the operator can always view the full system configuration even when all TV cameras are focused and zoomed on some particular subtask details. More on the SMSR task and control graphics visualization and the resulting graphics operator interface work can be found in Bejczy et al. (1990), Fiorini et al. (1993), Kim et al. (1991), Kim (1993).

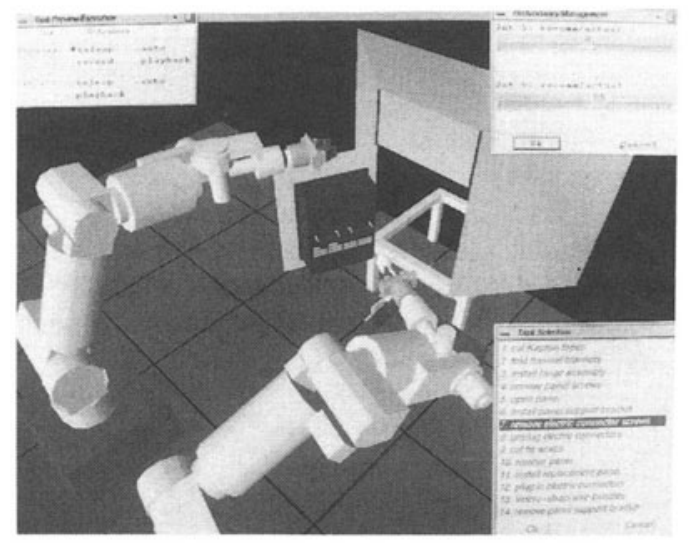

Figure 1. Graphics Operator Interface for Preview and OnLine Visualization, with Task Script Titles and Redundancy Mgmt. Status

Two major conclusions resulted from the SMSR VR task and control visualization off-line analysis: (i) The full task can only be performed with the given dual-arm setting if the base of either the dual-arm system or the task board (preferably the task board) is movable in two directions in a plane by about $\pm 45 \mathrm{~cm}$ in each direction and rotatable by about \pm 20 degrees, (ii) a small TV camera mounted to the base of the right arm's end effector would considerably contribute to the effectiveness and safety of the performance of several subtasks.

\section{VR calibration}

The actual utility of VR techniques in telerobotics to a high degree depends on the fidelity by which the graphic models represent the telerobot system, the task and task environment. A high fidelity VR calibration methodology has been developed by the ATOP project at JPL. This development has four major ingredients. First, creation of high-fidelity 3-D graphics models of remotely operated robot arms and objects of interest for robot arm tasks. Second, 
high-fidelity calibration of the 3-D graphics models relative to given TV camera 2-D image frames which cover the sight of both the robot arm and the objects of interest. Third, highfidelity overlaying of the calibrated graphics models over the actual robot arm and object images in a given TV camera image frame on a monitor screen as seen by the operator. Fourth, high-fidelity motion control of robot arm graphics image by using the same control software that drives the real robot.

The second ingredient is based on a TV camera calibration technique, using the Newtonian ideal pinhole model for image formation by the camera, which is a linear model. The camera calibration is performed by using the manipulator itself as a calibration fixture. The operator enters the correspondence information between 3-D graphics model points and 2-D camera image points of the manipulator to the computer. This is performed by repeatedly clicking with a mouse a graphics model point and its corresponding TV image point for each corresponding pair on a monitor screen which shows both the graphics model and the actual TV camera images. To improve calibration accuracy, several poses of the manipulator within the same TV camera view can be used to enter corresponding model and TV image points to the computer. Then the computer computes the camera calibration parameters. Because of the ideal pinhole model assumption, the computed output is a single linear $4 \times 3$ calibration matrix for a linear perspective projection.

Object localization is performed after camera calibration, entering corresponding object model and TV image points to the computer for different desired TV camera views. Again, the output is a single linear $4 \times 3$ calibration matrix for a linear perspective projection.

The actual camera calibration and object localization computations are carried out by a combination of linear and nonlinear least-squares algorithms, and the computational procedure depends upon whether an approximate initial solution is known.

After completing camera calibration and object localization, the graphics models of both the robot arm and the object can be overlaid with high fidelity on the corresponding actual images in a given TV camera view. The overlays can be in a wire-frame or solid-shaded polygonal rendering with varying levels of transparency, providing different visual effects to the operator for different task details. In a wire-frame format, the hidden lines can be removed or retained by the operator, dependent on the information needs in a given task.

These high fidelity fused virtual and actual reality displays became very useful tools for predicting and previewing robotic actions under communication time delay, without commanding and moving the actual robot hardware. The operator can generate visual effects of robotic motion by commanding the motion of the graphics image of the robot superimposed over TV pictures of the live scene. Thus, the operator can see the consequences of motion commands in real time, before sending the commands to the remotely located robot. This calibrated virtual reality display system can also provide high-fidelity synthetic or artificial TV camera views to the operator. These synthetic views make critical robot motion events visible that otherwise are hidden from the operator in a given TV camera view or for which no TV camera view is available. 


\section{VR performance demonstration}

The performance capabilities of the high-fidelity graphics overlay preview/predictive display technique described above was demonstrated on a large laboratory scale in May, 1993. A simulated life-size satellite servicing task was set up at the Goddard Space Flight Center (GSFC) in Maryland, and controlled 4000 kilometers away from the JPL ATOP control station. Three fixed TV camera settings were used at the GSFC worksite, and TV images were sent to the JPL control station over the NASA Select Satellite TV channel at video rate. Command and control data from JPL to GSFC and status and sensor data from GSFC to JPL were sent through the Internet computer communication network. The round-trip command/information time delay varied between four to eight seconds between the GSFC worksite and the JPL control station.

The task involved the exchange of a satellite module. This required inserting a $45 \mathrm{~cm}$ long power screwdriver, attached to a robot arm, through a $45 \mathrm{~cm}$ long hole to reach the module's latching mechanism at the module's backplane, unlatching the module from the satellite, connecting the module rigidly to the robot arm, and removing the module from the satellite. The placement of a new module back to the satellite's frame followed the reverse sequence of actions.

The experiments were performed successfully. The calibration and object localization errors at the critical tool insertion task amounted to $0.2 \mathrm{~cm}$ each, well within the allowed error tolerance. More on the VR calibration methodology and on the above-quoted experiments can be found in Kim et al. (1993) and in Bejczy et al. (1995). Figures 2 and 3 illustrate some of the overlay scenarios and a synthetic view.

\section{Visualization of the non-visible}

Recently a technique was developed for visualizing the highly constrained and complex geometric motion capabilities of a dual-arm system. The two arms work together on an object in a closed kinematic chain configuration. The motion space analysis has to take account of the base placement of the robot arms, object dimensions, object holding patterns and constraints. It turns out that this constrained motion space can be visualized as a complex 3-D object with hidden unreachable holes or cavities of varying shapes. The developed technique is an inverse computer vision procedure in the sense that it creates rather than recognizes visual forms. More on this technique can be found in Tarn et al. (1995).

Figure 4 shows a 3-D transparent view solution of a particular case problem, from different view angles. The upper left 3-D semi-transparent display is a perspective view such that the $\mathrm{x}$-axis is to the left, the $\mathrm{y}$-axis is to the right, and the z-axis is to the top. Two thick, black patches, seen to the left and right across the center are the unreachable spaces hidden in the outer light gray reachable space. The bigger transparent light gray object is the reachable space, and the lighter gray empty space parallel to the y-axis passing through the center corresponds to the forced unreachable space that avoids collision with robot bases. 


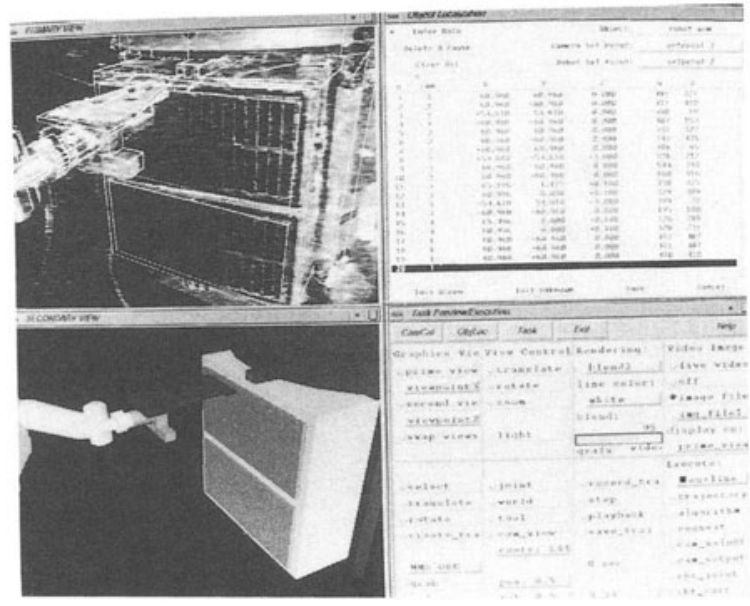

Figure 2. A Calibrated Graphics Overlay, with a Related Synthetic View.

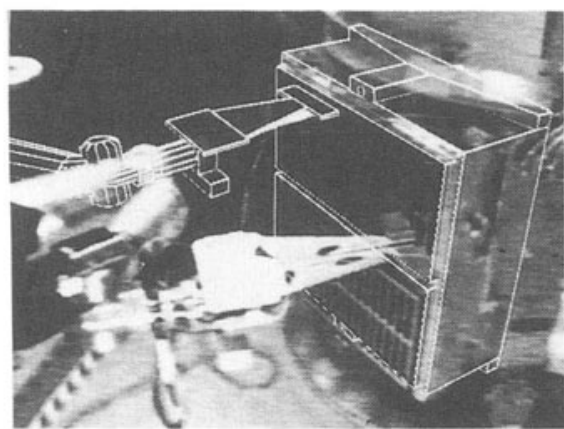

Figure 3a. Predictive/Preview Display of End Point Motion.

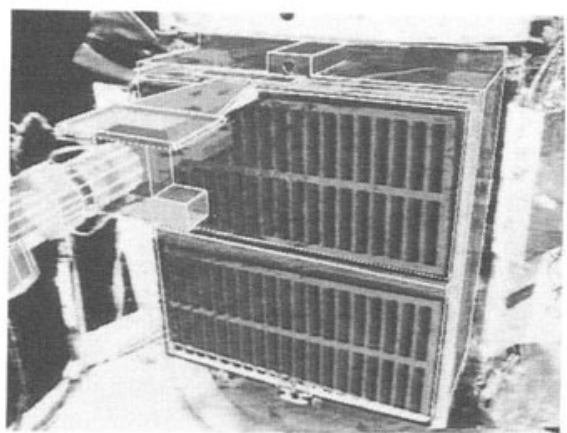

Figure 3b. Status of Predicted End Point after Motion Execution, for the Same Motion Shown in Figure 3a, but from Different Camera View. 


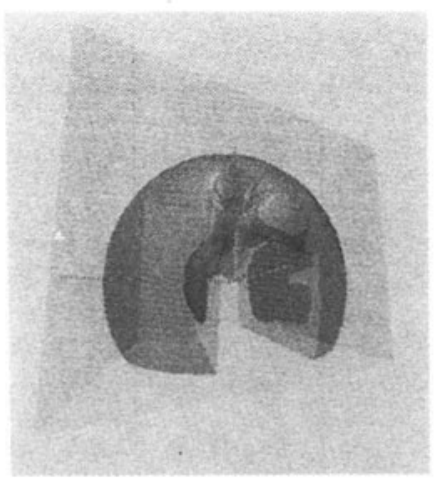

(a) View from $x-y$ direction

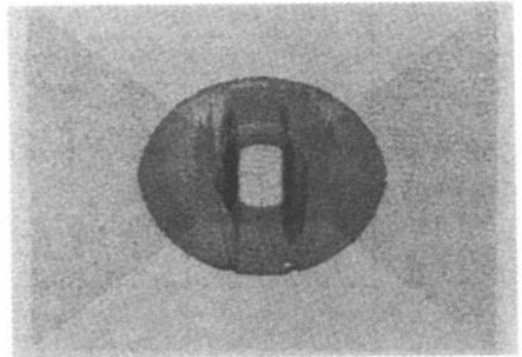

(c) View from $z$ direction

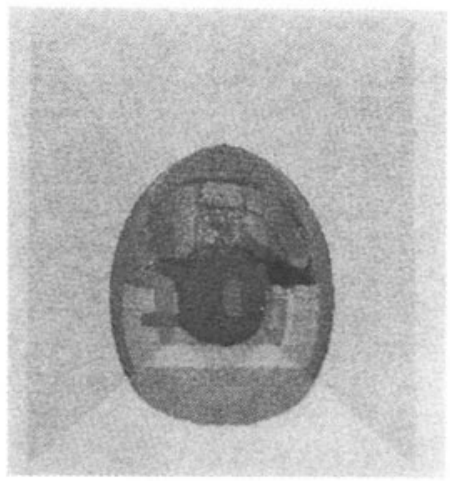

(b) View from $x$ direction

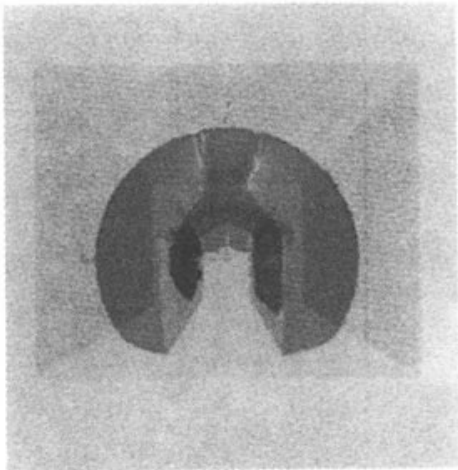

(d) View from y direction

Figure 4. Four views of the 3D transparent motion space for a rod of length $0.5 \mathrm{~m}$. 


\section{Virtual sensors}

The notion of 'virtual sensors' is referred to the simulation of sensors in a computer graphics environment that relate the simulated robot's interaction with the simulated objects. Two examples are quoted here: proximity sensing and contact force sensing.

Computer graphics simulation of proximity sensor signals is a relatively simple task since it only implies the computation of distance from a fixed point of a moving robot hand in a given (computed) direction to the nearest environment or object surface in the graphics 'world model'. For this simulation, the TELEGRIPTM software package of Deneb Robotics, Inc., has been adopted. The package provides an excellent interactive 3-D graphics simulation environment with CAD-model building, workcell layout, path designation, and motion simulation. It also provides various functions related to object distance computations and collision detection. TELEGRIP CLI (ascii-text Command Line Interpreter) commands include INQUIRE COLLISIONS (reporting collision and near miss status) and INQUIRE MINIMUM DISTANCE (reporting minimum distance between parts or devices). TELEGRIP GSL (Graphics Simulation Language) also supports ray cast ( ) function that computes the intersection distance from a point in a specified (ray cast) direction. Using these object distance computation functions, various proximity sensors can easily be simulated. More on this can be found in Bejczy et al. (1995).

Force/torque sensor can also be simulated by computing virtual contact forces and torques for given simulated geometric contact models. In general, an accurate simulation of virtual contact forces and torques can be very computation-intensive, but an approximate simulation, for example, a simplified peg-in-hold task, can be accomplished without difficulty, described in Bejczy et al. (1995). In this graphics simulation, the hole and its support structure are assumed to be rigid with infinite stiffness, while the robot hand holding the peg is compliant for all three Cartesian transitional axes and also for all Cartesian rotational axes. It is further assumed that the compliance center is located at a distance $\mathrm{L}$ from the tip of the peg with three lateral springs $\mathrm{k}_{\mathrm{x}}, \mathrm{k}_{\mathrm{y}}$, and $\mathrm{k}_{\mathrm{z}}$ and three angular springs $\mathrm{k}_{\mathrm{mx}}, \mathrm{k}_{\mathrm{my}}$, and $\mathrm{k}_{\mathrm{mz}}$. Both the operatorcommanded and the actual positions of the peg are described by the position of the compliance center. For a given operator-commanded peg position, the actual peg position after compliant accommodation can be different, depending upon the current state of the peg: whether the peg is currently in the hole or not. For the peg-not-in-hole state, two conditions are considered: no-touch or peg-on-wall. For the peg-in-hole state, four conditions are considered: no-touch, peg-side one-point contact, peg-tip one-point contact, or two-point contact.

Figure 5 shows a force-reflecting virtual reality training display for a peg-in-hole task. Contact forces and torques are computed and reflected back to a force-reflecting hand controller in real time. They are also displayed on the upper left corner of the display screen at $20 \mathrm{~Hz}$ rate. 


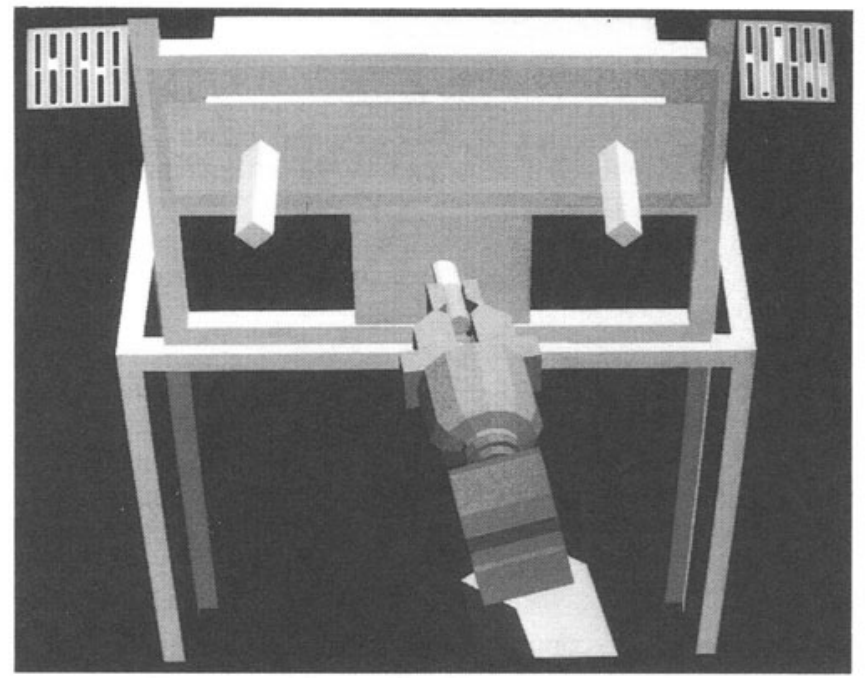

Figure 5. Force-Reflecting VR Training Display; Simulated Contact Force/Moment Values Shown in the Upper Left Bar Display.

\section{VIRTUAL REALITY AND MANUFACTURING}

From an engineering viewpoint, manufacturing as a technological activity comprises four classes of engineering task: product design, system set-up design for production process, actual set-up of production system, and operation of the actual production system. VR techniques, connected to CAD data banks, can substantially contribute to the success and cost-effectiveness of all four classes of manufacturing engineering tasks in a very efficient way. The efficiency of VR techniques applied to manufacturing is rooted to two basic capabilities of VR methods: high-fidelity visualization providing model and situation representation in the 3-D space and the power of interactive handling of large data sets in real time or near real time.

\section{Product design}

VR techniques offer the opportunity to expand the product design capabilities of CAD techniques by new technical dimensions to satisfy ergonomic, aesthetic, assembly, customization and quick prototyping considerations and requirements. In fact, VR techniques connected to CAD data banks can elevate product simulation to or near to a proof-of-concept level, saving considerable time and cost.

Automobile manufacturers already capitalized on these capabilities very successfully during the past five to six years. To keep the volume of this article within limits, only one advanced 
example is quoted here. Kalawski (1993) reports the efforts of researchers at the British Aerospace Brough Laboratory. They created a Ruver 400 model car interior by using CADlike objects and a graphical programming language. The system included a high-resolution HRX EyePhone, a DataGlove, a Convolotron for 3-D sound, and a set of SGI computers for graphics rendering. To reinforce realism of VR simulation, the researchers used a real car seat. Therefore, the designers were able to study the ergonomics of the car interior accurately and change the position and orientation of virtual parts when needed. It was then possible to redesign the interior of the car while immersed in the graphics simulation environment. For example, the designers could grab and move the steering wheel assembly from the "right-hand" drive position of the UK, Japan and Portugal to the "left-hand" drive position of the US and the rest of the European countries.

\section{Production system design}

A production system consists of several passive and active cooperating devices, parts to be treated, moved and assembled, a logical sequence of actions, a nominal time line of actions, and the capability of recovering from a variety of possible errors. Moreover, it is often required to design a production system with some flexibility in mind relative to changes in the product to be produced by the system. A flexible manufacturing system typically also employs robots. VR techniques, connected to CAD data banks, offer very valuable opportunities and capabilities not only for the design but also for the real-time simulation, control and monitoring of flexible robotic manufacturing systems. Good examples of VR simulation of flexible manufacturing cells are described in Apostoli et al. (1993), Apostoli et al. (1995) and Moran et al. (1995). These quoted examples also cover some programming, control and monitoring aspects of a manufacturing cell using VR simulation which gives 3-D representation of the global geometry and of the changing geometric relations in a working cell.

An other good example is reported by Strommer et al. (1993), using a transputer network for development of control of robots in a manufacturing environment. In the reported effort, the computer control programming is done on a virtual robot and its virtual environment. The environment can be a manufacturing cell, and utilizing all available feedback elements of the human-machine interface. The interface is divided into a human-computer module and a connection to the real world which contains a robot of the same type as the virtual robot. The information loop between computer and real world is then closed by sensor data coming from the real world. After control code development and debug, the code is stored and downloaded to the real robot which then executes in on the real environment.

\section{Production system operation}

First of all, the operation of computer controllable production systems poses a complex programming problem. There are some commercially available software products that can help develop programming of production systems using VR methods. Examples are the IGRIPTM and TELEGRIPTM software packages of DENEB Robotics, Inc. (P.O. Box 214687, Auburn Hills, MI 48321-4687, USA; Internet address: support@deneb.com). These products use open architecture, industry standards and network protocols, and offer application-specific options. 
They also demonstrated meeting acceptance criteria imposed by automotive companies and functioning in real time.

An interesting and quite active research theme today is the programming of assembly tasks by recognizing human teaching operations. Examples can be found in Takahashi et al (1992), Tso et al. (1995) and Tung et al (1995). This type of programming approach also can be elevated to higher levels and generalized as outlined in a system description by Ogata et al. (1996). That system operates in two phases: the teaching phase, and the execution phase. In the teaching phase, the system learns a task in a specific environment from a human operator using a VR teaching interface, then translates it to a general task description in the task interpreter, and stores it. In the execution phase, the system retrieves the general task description, observes the environment where the task will be executed, modifies the learned motion in the task planner, and executes the task.

\section{CONCLUSION}

Manufacturing appears to be a major beneficiary of VR technology and of its evolving capabilities in many ways. It can considerably improve design and production processes and introduce new approaches to the programming style of computer controlled manufacturing systems, rendering the programming effort more efficient and more transparent.

The application of VR technology in manufacturing is not yet a fully evolved capability and accepted practice, and it requires some investment in proper education and teaching, and in appropriate commercially available hardware/software tools. The cost of appropriate commercial tools is on a decreasing trend as we know it from the price history of the last few years. The real progress and success will depend on human attitude and education which in turn calls for an effective teaming of VR and manufacturing technologists.

\section{REFERENCES}

Apostoli, R.S., Moran, D.D., Trombotto, G.D., Abate, F.J. (1993), A wholly designed and built flexible manufacturing cell (FMC) for training and research purposes. 12th Brazilian Congress of Mechanical Engineers; Brazilia, Brazil; pp. 1603-1606.

Apostoli, R.S., Luciano, C.J. (1995), Manufacturing Simulation in a flexible system. 4th Symposium on Low Cost Automation; (IFAC-AADECA); Bs. As., Argentina; pp. $446-451$.

Bejczy, A.K., Kim, W.S., Venema, S. (1990), The phantom robot: predictive displays for teleoperation with time delay; Proc. IEEE Int'l. Conf. on Robotics and Automation, Cincinnati, $\mathrm{OH}$. 
Bejczy, A.K., Kim, W.S. (1995), Sensor fusion in telerobotic task controls; Proc. IEEE Int'l Conf. on Intelligent Robots and Systems, Pittsburgh, PA.

Fiorini, P., Bejczy, A.K., Schenker, P.S. (1993), Integrated interface for advanced teleoperation; IEEE Control Systems Magazine, Vol. 13, No. 5.

Kalawski (1993), The Science of Virtual Reality and Virtual Environments; Addison-Wesley Publ. Co., U.K., 405 pp.

Kim, W.S., Bejczy, A.K. (1991), Graphics displays for operator aid in telemanipulation; Proc. IEEE Int'l. Conf. on Systems, Man, an Cybernetics, Charlottesville, VA.

Kim, W.S. (1993), Graphical operator interface for space telerobotics; IEEE Int'l. Conf. on Robotics and Automation, Atlanta, GA.

Kim, W.S., Bejczy, A.K. (1993), Demonstration of a High Fidelity Predictive/Preview Display Technique for Telerobotic Servicing in Space; IEEE Transactions on Robotics and Automation, Special issue on space robotics (October).

Moran, O.D., Rosales, A.G., Luciano, C.J. (1995), Real-time control of a tutorial flexible manufacturing cell; 4th Symposium of Low Cost Automation; (IFAC-AADECA); Bs. As. Argentina; pp. 311-315.

Ogata, H., Tsuda, M., Mizukawa, M. (1996), Teaching 6-dot tasks for robot operating assembly in the same category; to appear in Proc. 8th Int'l. Conf. on Advanced Robotics, Monterey, CA.

Strommer, W., Neugebauer, J., Flaig, T. (1993), Transputer-based workstation as implemented for the example of industrial robot control; Proc. of Interface to Real and Virtual Words Conference, Montpellier, France; pp. 137-146.

Takahashi, T., Ogata, H. (1992), Robotic assembly operation based on task-level teaching in virtual reality; Proc. IEEE Int'l. Conf. on Robotics and Automation; pp. 1083-1088.

Tso, S.K., Liu, K.P. (1995), Automatic generation of robot programming codes from perception of human demonstration; Proc. IEEE Conf. on Intelligent Robots and Systems; pp. 23-28.

Tung, C.P., Kak, A.C. (1995), Automatic learning of assembly tasks using a DataGlove system; Proc. IEEE Conf. on Intelligent Robots and Systems; pp. 1-8. 
Acknowledgment: This work was carried out at the Jet Propulsion Laboratory, California Institute of Technology, under contract by the National Aeronautics and Space Administration (NASA).

\section{Brief Author Biography}

Bejczy, Antal K., is a Senior Research Scientist at the Jet Propulsion Laboratory, Pasadena, CA, and an Affiliate Professor, Department of Systems Science and Mathematics, Washington University, St. Louis, MO. He received B.S. in EE at the Technical University of Budapest, Hungary; M.S. in Natural Sciences, University of Oslo, Norway; Ph.D. in Applied Physics, University of Oslo, Norway in 1963. He was a Research Fellow and Senior Resident Research Associate at the California Institute of Technology in 1966-1969, at the Dept. of Applied Sciences. He joined JPL in 1969. First, he worked on advanced space mission studies, then turned his interest on robotics and teleoperation in 1972. He published about 150 journal and conference papers mainly on topics in dynamic modeling, sensing, control, and humanmachine interaction in telerobotics, including telepresence and virtual reality; 12 chapters in various edited volumes in robotics, and co-edited one book on "Parallel Computation Systems for Robotics." He was president of the IEEE Council on Robotics and Automation in 1987, and serves as an ADCOM member in the IEEE Robotics and Automation Society since 1990. He organized numerous special sessions on robotics and teleoperation at IEEE conferences. He became an IEEE Fellow in 1987. He received the NASA Exceptional Service Medal in 1991. He received over 40 NASA Technical Innovation awards, and holds 6 U.S. patents. He is General Chairperson of the "8th International Conference on Advanced Robotics" to be held in July, 1997. 